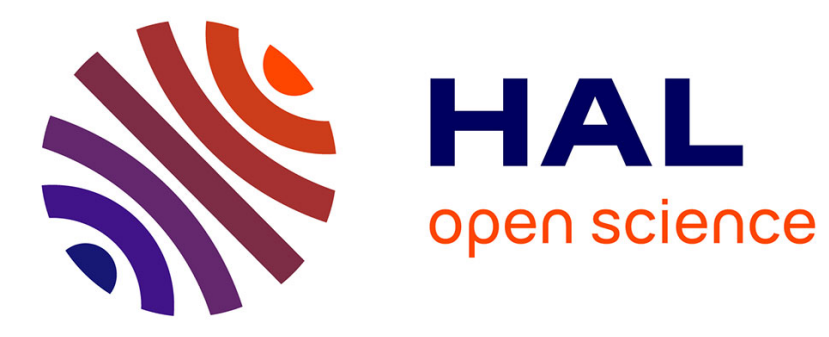

\title{
Preferential concentration of heavy particles in turbulence
}

\author{
Martin Obligado, Tomás Teitelbaum, Alain H Cartellier, Pablo Mininni, \\ Mickaël Bourgoin
}

\section{- To cite this version:}

Martin Obligado, Tomás Teitelbaum, Alain H Cartellier, Pablo Mininni, Mickaël Bourgoin. Preferential concentration of heavy particles in turbulence. Journal of Turbulence, 2014, 15 (5), pp.293-310. 10.1080/14685248.2014.897710 . hal-01924958v2

\section{HAL Id: hal-01924958 \\ https://hal.science/hal-01924958v2}

Submitted on 5 Jun 2020

HAL is a multi-disciplinary open access archive for the deposit and dissemination of scientific research documents, whether they are published or not. The documents may come from teaching and research institutions in France or abroad, or from public or private research centers.
L'archive ouverte pluridisciplinaire HAL, est destinée au dépôt et à la diffusion de documents scientifiques de niveau recherche, publiés ou non, émanant des établissements d'enseignement et de recherche français ou étrangers, des laboratoires publics ou privés. 


\title{
Preferential Concentration of Heavy Particles in Turbulence
}

\author{
Martín Obligado $^{\mathrm{a}}$, Tomás Teitelbaum ${ }^{\mathrm{b}}$, Alain Cartellier ${ }^{\mathrm{c}}$, Pablo Mininni ${ }^{\mathrm{b}}$ and \\ Mickaël Bourgoin ${ }^{\mathrm{C} * *}$ \\ a Turbulence, Mixing and Flow Control Group, Department of Aeronautics, Imperial \\ College London, London SW7 2AZ, United Kingdom \\ b Departamento de Física, Facultad de Ciencias Exactas y Naturales, Universidad de \\ Buenos Aires and IFIBA, CONICET, Ciudad Universitaria, 1428 Buenos Aires, \\ Argentina \\ ${ }^{\mathrm{c}}$ Laboratoire des Écoulements Géophysiques et Industriels, CNRS/UJF/G-INP, \\ UMR5519, Université de Grenoble, BP53, 38041, Grenoble Cedex 09, France
}

Published in

Martn Obligado, Toms Teitelbaum, Alain Cartellier, Pablo Mininni \& Mickal Bourgoin (2014) Preferential concentration of heavy particles in turbulence, Journal of Turbulence, 15:5, 293-310, DOI: 10.1080/14685248.2014.897710

\begin{abstract}
Particle laden flows are of relevant interest in many industrial and natural systems. When the carrier flow is turbulent, a striking feature is the phenomenon called preferential concentration: particles denser than the fluid have the tendency to inhomogeneously distribute in space, forming clusters and depleted regions. We present an investigation of clustering of small water droplets in homogeneous and isotropic active-grid-generated turbulence. We investigate the effect of Reynolds number $\left(R_{\lambda}\right)$ and Stokes number $(S t)$ on particles clustering in the range $R_{\lambda} \sim 200-400$ and $S t \sim 2-10$. Using Voronoï diagrams, we characterize clustering level and clusters properties (geometry, typical dimension and fractality). The exact same Voronoï analysis is then applied to investigate clustering properties of specific topological points of the velocity field of homogeneous isotropic turbulence obtained from Direct Numerical Simulations at $R_{\lambda} \sim 220$ and 300. The goal is to compare clustering properties of actual particles with those of such points in order to explore the relevance of possible clustering mechanisms, including centrifugal effects (heavy particles sampling preferentially low vorticity regions) and sweep-stick mechanisms (heavy particles preferentially sticking to low acceleration points). Our study points toward a leading role of zero-acceleration points and sweep-stick effects, at least for the experimental conditions considered in this study.
\end{abstract}

\section{Introduction}

A striking feature of turbulent flows laden with inertial particles is the so-called preferential concentration phenomenon which leads to particles clustering with very strong inhomogeneities in the concentration field at any scale. This has now been widely observed in many experimental and numerical configurations including homogeneous and isotropic turbulence [19, 4, 18]. A robust result is that the concentration field is more intermittent for particles whose Stokes number (defined as the ratio of the particle relaxation time to the Kolmogorov viscous time) is close to unity. Aliseda and co-workers [1] have suggested that the local enhancement of the concentration could be responsible for the measured enhancement of particles settling velocity in turbulent flows due to collective effects. More generally preferential concentration eventually affects the dynamics and the interactions of particles in a turbulent field, with possible impact on important phenomena as for instance collisions, coalescence and fragmentation, phase transitions, etc. The problem is therefore relevant both, from the fundamental aspect of particle/turbulence interaction

*Corresponding author. Email: mickael.bourgoin@legi.cnrs.fr 
and also in the context of practical issues, such as rain initiation, spray dynamics, reactive dispersed flows, etc.

The usual physical interpretation of preferential concentration of dense particles in turbulence relies on the centrifugal expulsion of heavy particles from the core of turbulent eddies, hence concentrating the particles in low-vorticity regions of the carrier flow. During the past few years, Vassilicos and coworkers proposed an alternative scenario, based on a stick-sweep mechanism [8], where particles tend to stick preferentially to zero-acceleration points of the carrier flow with which they are then advected. Using a simple point particle model for the particle's equation of motion (in the limit where only the drag term is preserved in the Maxey-Riley-Gatignol equation [10, 5]) Coleman et al. [3] have shown that the centrifugal scenario is likely to prevail for particles with Stokes number less than unity, while sweep-stick is expected to be dominant for Stokes number above unity.

In the present article, we address the question of the physical origin of preferential concentration of small heavy particles in turbulence. The mechanisms described above suggest the existence of specific correlations of particles position with prescribed regions of the carrier flow (low vorticity points in the centrifugal scenario, or zero-acceleration points in the sweep-stick approach). Ideally, the experimental investigation of these mechanisms would therefore require to measure simultaneously the dispersed phase (the particles) and the continuous phase (the carrier flow), in order to access these correlations. Such experiments are still very challenging to be achieved and very few such simultaneous measurements have yet been attempted (the work by Poelma et al. [16] is a pioneering example of both the complexity and the richness of such measurements). In the present study we propose a different strategy, consisting in comparing direct numerical simulations (DNS) of the continuous phase with experimental measurements of the dispersed phase (hence avoiding approximations intrinsic to usual numerical models for the equation of motion of inertial particles), and to compare the clustering properties of real particles in the experiment with those of specific topological points of the flow in the DNS.

The article is organized as follows. In section 2 we describe the experimental investigation of preferential concentration of inertial particles (small water droplets) in homogeneous and isotropic turbulence generated downstream an active grid in a wind tunnel. The details of the experiment are given before analyzing clustering properties using Voronoï diagrams [2]. Main trends with Reynolds and Stokes numbers are briefly discussed. Section 3 is then dedicated to the investigation of clustering properties of specific points of a homogeneous and isotropic turbulent field obtained from DNS. A close comparison with the case of actual particles in the experiment is proposed. Finally, section 4 summarizes the main findings and draws the main conclusions of this study.

\section{Experimental study: preferential concentration of inertial par- ticles in turbulence}

\subsection{Experimental Setup}

Experiments are conducted in a wind tunnel with a measurement section $4 \mathrm{~m}$ long and a square crosssection of $0.75 \times 0.75 \mathrm{~m}^{2}$ (see figure 1). Turbulence is generated with an active grid made of 16 rotating axes (eight horizontal and eight vertical) mounted with co-planar square blades. Each axis is driven by an independent step motor with random rotation rate and direction, which are both changed randomly in time. Active grids with such random protocols are known to produce higher turbulence levels than classical passive grids, with still good homogeneity and isotropy properties $[9,14,17]$. The mean velocity of the wind varies from $3.4 \mathrm{~m} / \mathrm{s}$ up to $7.6 \mathrm{~m} / \mathrm{s}$, corresponding to a range of Reynolds number (based on Taylor micro-scale) $R_{\lambda} \in[230 ; 400]$. Table 1 summarizes the main turbulence parameters of the flow generated at the measurement volume location (3 $\mathrm{m}$ downstream the active grid) for the 6 mean wind velocities investigated.

As inertial particles we use small water droplets generated by 36 high-pressure atomizers (distributed on a $6 \times 6$ mesh with identical spacing than the grid) located in a transverse plane $15 \mathrm{~cm}$ downstream the grid. The droplets size distribution (measured with a Spraytec diffractometer from Malvern Instruments Ltd) is peaked around a most probable droplet diameter of the order of $D_{p} \sim 50 \mu \mathrm{m}$, but is relatively polydisperse (the standard deviation of particles diameter is $\sigma_{D_{p}} \simeq 35 \mu \mathrm{m}$ ). We have checked that this size distribution is robust and does no depend significantly on the mean wind velocity. Particles therefore always have a sub-Kolmogorov size (see table 1). The volume fraction of water droplets is in the range $\phi_{v}=\left[5 \cdot 10^{-5}-2 \cdot 10^{-4}\right]$ (the lower the average wind velocity, the larger the volume fraction). We define the droplets Stokes number as the ratio $S t=\tau_{p} / \tau_{\eta}$, with $\tau_{\eta}$ the carrier flow dissipation scale estimated from hot-wire anemometry (see table 1 ) and $\tau_{p}$ the particles viscous relaxation time estimated for the 


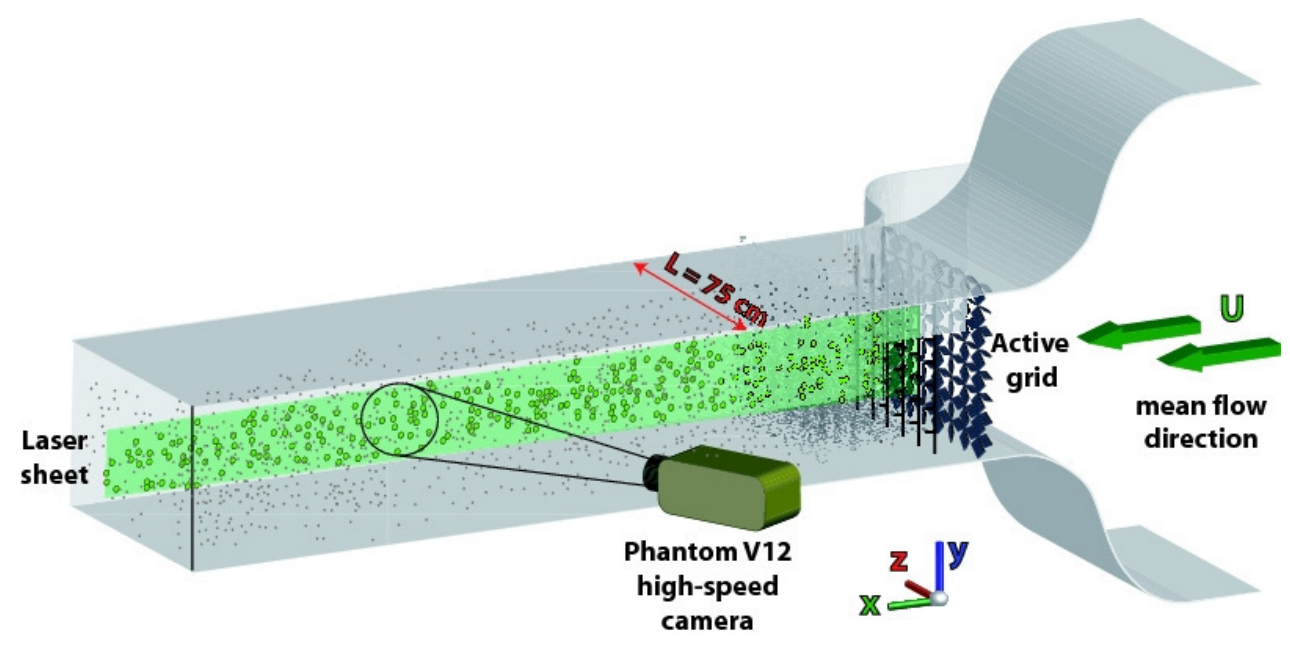

Figure 1: Sketch of the experimental setup.

most probable particle diameter as $\tau_{p}=\frac{\rho_{p} D_{p}^{2}}{18 \rho_{a i r} \nu}$, with $\rho_{p}$ the particle density, $\rho_{a i r}$ and $\nu$ the density and kinematic viscosity of air at working temperature. The Stokes number can be equivalently estimated as $S t=\left(D_{p} / \eta\right)^{2} \Gamma / 18$ with $\eta$ the dissipation scale of the carrier turbulence and $\Gamma=\rho_{p} / \rho_{\text {air }}$, which in the present case (water particles carried in air) simply gives $S t \simeq 46\left(D_{p} / \eta\right)^{2}$. Therefore, as the most probable particle diameter $D_{p}$ is kept constant and does not depend on flow conditions, varying the carrier flow Reynolds number (and hence varying the dissipation scale $\eta$ ) also results in a variation of the particles Stokes number.

Particles are illuminated with a laser sheet with millimetric width. We use an 8W Copper laser pulsed at $10 \mathrm{kHz}$. Acquisitions are performed using a Phantom V12 high speed camera operated at $10 \mathrm{kHz}$ (synchronized with laser pulses) and acquiring 12 bits images at a resolution of 1280 pixels $\times 488$ pixels. The camera is mounted with a $90 \mathrm{~mm}$ macro lens, giving a measurement window of $125 \mathrm{~mm}$ (along $x$ ) $\times 55 \mathrm{~mm}$ (along $y$ ), though homogeneous illumination conditions (tested a posteriori during the post-processing) were actually limited to a smaller visualization window of $70 \mathrm{~mm}$ in the streamwise $x$-direction and $50 \mathrm{~mm}$ in the transverse $y$-direction. Therefore, the explored domain covers a significant fraction of the integral scale of the carrier flow in each direction. Images are taken in a slight forward scattering configuration, in order to improve the contrast of particles images. The view angle with respect to the laser sheet is then of the order of $60^{\circ}$, requiring a Scheimpflug mount to compensate the loss of depth of field. At the working spatial resolution and repetition rate, the onboard memory of our camera $(8 \mathrm{~Gb})$ allows to record slightly more than $10^{4}$ images (hence slightly more than one second of recording), what corresponds already to a few integral time scales of the carrier turbulence. For each experimental configuration we acquire at least 15 such recordings ; thus a set of more than $1.5 \cdot 10^{5}$ images are obtained for each experiment. In the present work we only consider a subsample of about $10^{4}$ statistically independent images. Particles are then detected on each image, following standard thresholding and center finding algorithms [15].

\begin{tabular}{|c|c|c|c|c|c|}
$R e_{\lambda}$ & $U(\mathrm{~m} / \mathrm{s})$ & $L(\mathrm{~cm})$ & $\eta(\mu \mathrm{m})$ & $\epsilon\left(\mathrm{m}^{3} \mathrm{~s}^{-3}\right)$ & $S t$ \\
\hline \hline 234 & 3.4 & 13.0 & 280 & .69 & 2.1 \\
264 & 4.0 & 13.2 & 240 & 1.2 & 3.3 \\
295 & 4.8 & 13.5 & 208 & 2.0 & 4.3 \\
331 & 5.7 & 13.8 & 178 & 3.4 & 5.8 \\
357 & 6.4 & 14.0 & 160 & 4.7 & 6.6 \\
400 & 7.6 & 14.3 & 140 & 7.7 & 9.9
\end{tabular}

Table 1: Experimental parameters : Reynolds number based on Taylor microscale $\left(R_{\lambda}\right)$, mean wind velocity $(U)$, energy injection scale $(L)$, dissipation scale $(\eta)$, energy dissipation rate per unit mass $(\epsilon)$, Stokes number $(S t)$. 


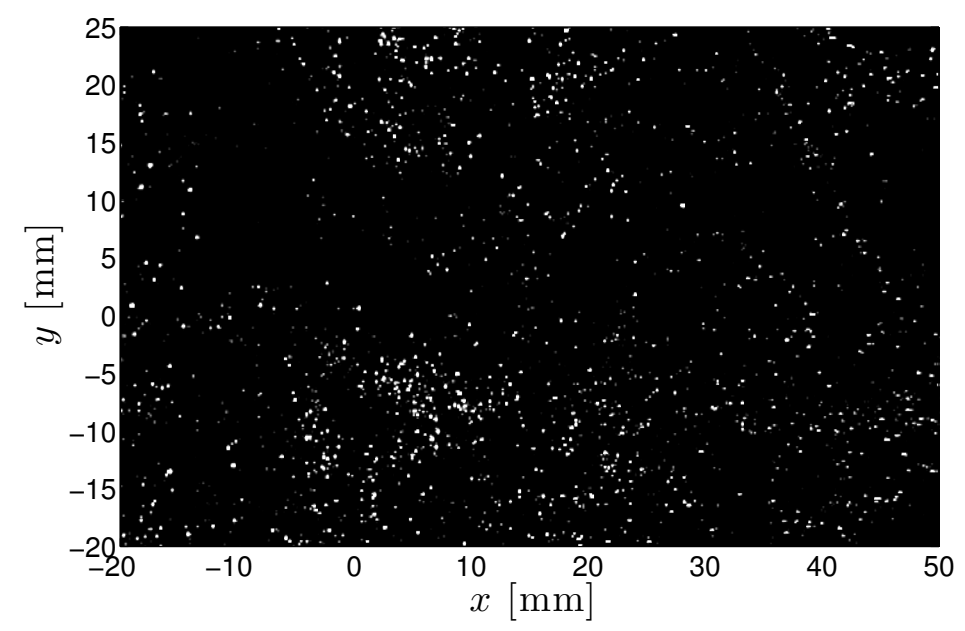

(a)

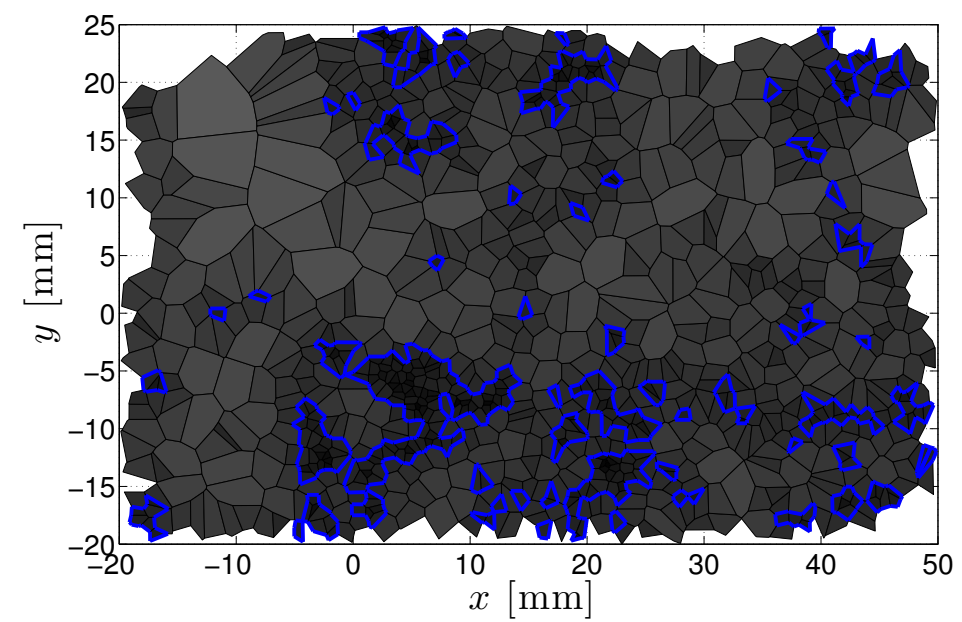

(b)

Figure 2: (a) Example of raw image of water droplets in the active grid generated turbulence. (b) Corresponding Voronoï diagram and detected clusters. 


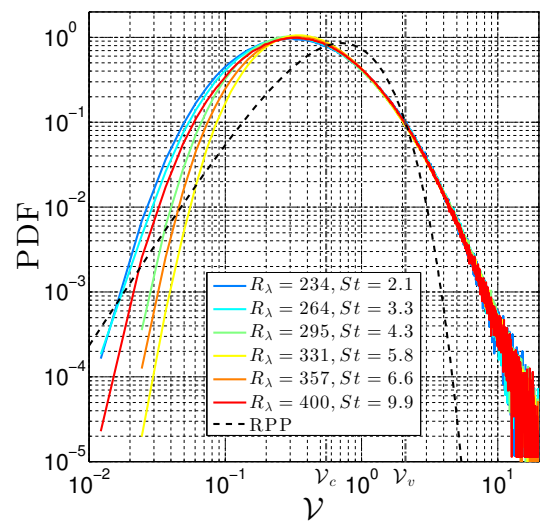

(a)

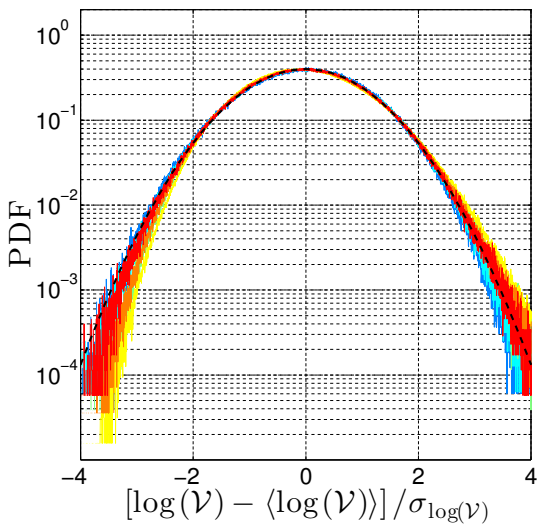

(b)

Figure 3: (a) Probability distribution of normalized Voronoï area $\mathcal{V}$ for experiments at different Reynolds number and Stokes number. The red dashed line represents a random Poisson process (RPP) distribution. (b) Probability distribution function, centered and reduced, of $\log (\mathcal{V})$ for the same experiments as in figure (a). The black dashed line represents a Gaussian distribution.

\subsection{Clustering evidence}

In order to diagnose and quantify the clustering properties of the inertial droplets in the grid generated turbulence we use a Voronoï tessellation [2] analysis of particles center. This method was first introduced in the field of particles in turbulence by Monchaux et al. $[12,13]$ who showed the relevance of this approach to investigate clustering of particles from $2 \mathrm{D}$ images in a laser sheet; it was recently extended to 3D measurements by Tagawa et al. [20]. Figure 2 shows an example of raw image of the particles in our experiment, and its corresponding Voronoï diagram. The analysis of the statistics of the area $\mathcal{A}$ of Voronoï cells (whose inverse corresponds to the local concentration of particles) has been shown to be particularly relevant to address preferential concentration issues [13]. We follow here this approach.

Probability density functions (PDFs) of normalized Voronoï cell areas $(\mathcal{V}=\mathcal{A} /\langle\mathcal{A}\rangle)$ and their standard deviation $(\sigma \mathcal{V})$ from this same set of experiments were analyzed in a previous work by Obligado et al. [15]. The main results of this previous analysis are recalled in figures 3a\&b and 4. In figure 3a the strong deviation between the PDFs of normalized Voronoï areas of water droplets in the experiment for different Reynolds number (colored lines), and that of a random Poisson process (RPP) reveals the preferential concentration phenomenon: large areas (depleted regions) and small areas (concentrated regions) are statistically over-represented for the case of inertial particles. As in Monchaux et al. it was found that (i) Reynolds and Stokes number effects essentially influence small and highly concentrated areas (left tail of the PDFs in figure 3a) while large and depleted areas (right tail) remain independent of Reynolds and Stokes numbers and (ii) the centered-reduced PDFs of $\log (\mathcal{V})$ are well approximated by a Gaussian distribution, suggesting that Voronoï area distributions are close to lognormal. Finally, Figure 4 shows how $\sigma_{\mathcal{V}}$ varies with the particle Stokes number St. We recall that the standard deviation of twodimensional Voronoï cells for a RPP can be obtained analytically, being $\sigma_{\mathcal{V}}^{R P P}=0.53$. Higher values of $\sigma_{\mathcal{V}}$ indicate the presence of clustering, and the higher $\sigma_{\mathcal{V}}$ the stronger the clustering. Figure 4 shows that clustering in active grid experiments is significantly more intense than what was observed in previous measurements by Monchaux et al. [12] at lower Reynolds numbers. Interestingly, though $\sigma_{\mathcal{V}}$ changes by less than $10 \%$ over the different active grid experiments, the present data set shows a local maximum of clustering for $S t \sim 3-4$ (corresponding to the experiment at $R_{\lambda} \sim 300$ ). Although a deeper insight would require to disentangle Stokes and Reynolds number effects, these trends are consistent with those reported by Monchaux et al. [12] at lower Reynolds numbers. The overall effect of increasing $R_{\lambda}$ (compared to Monchaux et al. [12]) is clearly to increase the clustering level (quantified by $\sigma_{\mathcal{V}}$ ). Hence, if we take for instance the point at $S t \simeq 4$ (corresponding to $R_{\lambda} \simeq 300$ ) as a reference, one would expect that for the evolution of $\sigma_{\mathcal{V}}$ as a function of Stokes keeping constant $R_{\lambda}$ at 300, points at the right of the reference point (for $S t>4$ ) will be actually below the points (stars) in figure 4 (which were obtained for larger $R_{\lambda}$ ), while points at the left of the reference point (for $S t<4$ ), will be above the points (stars) in 


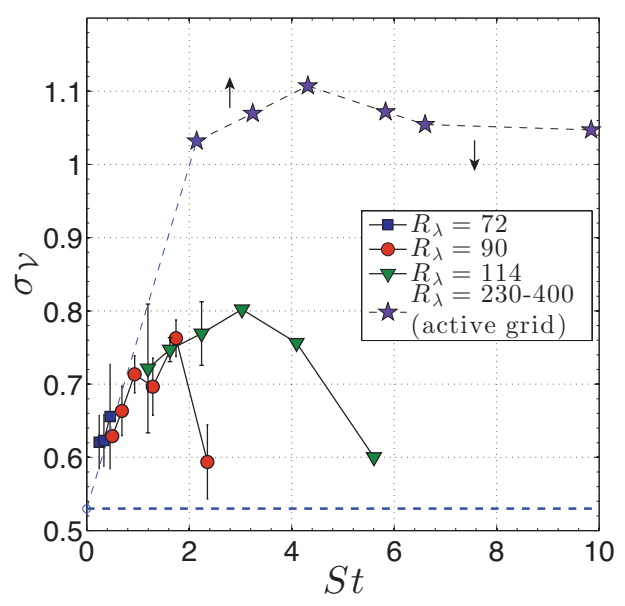

Figure 4: Evolution of the standard deviation of the normalized Voronoï area with Reynolds number. The arrows indicate how the dashed-line curve is be expected to be changed if the Stokes number was varied keeping $R_{\lambda}$ constant around 300 (what corresponds in the present experiment to the point at $S t \simeq 4$ ).

figure 4 (which were obtained for smaller $R_{\lambda}$ ). This is emphasized by the arrows represented in figure 4 . Therefore, it is likely that the curve representing the evolution of $\sigma_{\mathcal{V}}$ as a function of Stokes at a constant $R_{\lambda}$ of 300 should look more peaked than the dahed-line curve represented in figure 4 (which mixes points at different $R_{\lambda}$ ) with a maximum of clustering for an optimal Stokes below 4 .

\subsection{Clusters geometry}

The previous study has confirmed the presence of clustering and its dependency both on Stokes and Reynolds numbers. We focus now on the characterization of the geometry and the structural properties of clusters.

Identifying clusters. This requires first to identify clusters as individual entities. We follow the protocol proposed by Monchaux et al. [12]: normalized Voronoï areas smaller than the mean which are over-represented compared to a RPP are defined as belonging to clusters (equivalently, Voronoï cells larger than the mean which are over-represented compared to a RPP are defined as belonging to voids). In other words cells whose area is below the threshold defined by the intersection $\mathcal{V}_{c}$ (see figure 3a) belong to clusters, while cells above the intersection $\mathcal{V}_{v}$ belong to voids (see figure $3 \mathrm{a}$ ). Clusters are then defined as the ensembles of connected clusters with $\mathcal{V}<\mathcal{V}_{c}$. Figure $2 \mathrm{~b}$ shows an example of identified clusters. In the sequel we present the results of the statistical analysis of clusters properties (voids, which are not discussed here present very similar geometrical properties compared to clusters, although naturally at larger scales).

Statistics of clusters area. Figure 5a shows the PDFs of the area $A_{C}$ of clusters, normalized by the average area $\left\langle A_{C}\right\rangle$. Interestingly, all PDFs collapse onto a single curve. As Monchaux et al. [12], we find that for large areas the PDFs of clusters area follow a power law with an exponent close to -2 . However, contrary to Monchaux et al., we do observe a clear maximum of these PDFs, with a peak around a most probable area $A_{C}^{\max } \simeq 0.2\left\langle A_{C}\right\rangle$. This gives strong experimental evidence that clusters do have a typical characteristic area, in agreement with the earlier work by Aliseda et al. [1]. We can note at this point that the conclusion on the absence of a typical cluster size drawn by Monchaux et al. [12] was essentially motivated by the -2 power law of the clusters area PDF (a purely algebraic PDF with a -2 exponent does not even have a converged average). However, a closer look at figure 7a in [12] shows that the data of Monchaux et al. is not purely algerbriac, but presents a clear concave bending for the smallest areas of detected clusters, which is not incompatible with a maximum of clusters area PDF (of the order of a few millimeters squared). Repeating experiments in the range of moderate Reynolds numbers explored by Monchaux et al. may be interesting in order to clarify this point. However, the data in the range of parameteres explored in the present work points unambiguosuly towards the existence of a typical cluster size. 


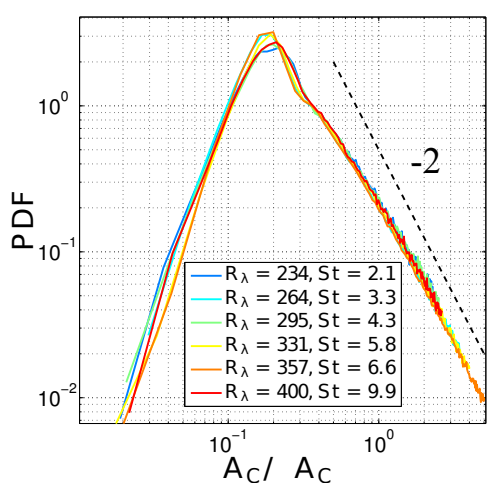

(a)

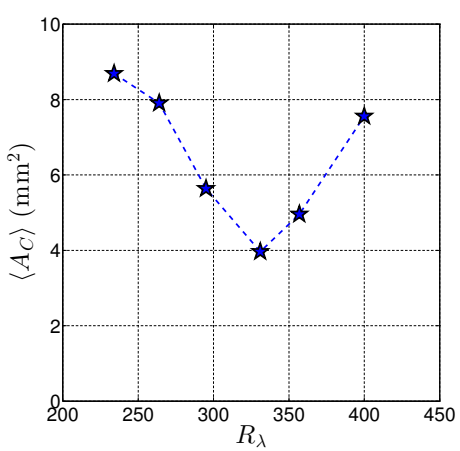

(b)

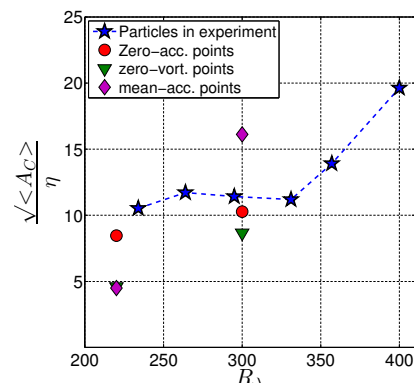

(c)

Figure 5: (a) PDFs of clusters area normalized by the mean. (b) Mean value of clusters area as a function of $R e_{\lambda}$. (c) Linear dimension of clusters normalized by the dissipation scale of the flow. Red circles represent the value obtained numerically in section 3 for clusters of zero-acceleration points, the green square is for clusters of zero-vorticity points and the magenta diamond for clusters of mean-acceleration points.

Typical size of clusters. Figure $5 \mathrm{~b}$ shows how the mean value $\left\langle A_{C}\right\rangle$ of clusters area varies with $R_{\lambda}$ (or equivalently with $S t$, as it ought to the relation $S t=46\left(D_{p} / \eta\right)^{2}$ previously discussed). The typical area is found of the order of a few squared millimeters, with a minimum around $R_{\lambda}=331$ (or $S t=5.8$ ), slightly larger than the corresponding maximum for the standard deviation of Voronoï cells in figure 3c. Figure $5 \mathrm{c}$ shows the typical linear dimension $\sqrt{\left\langle A_{C}\right\rangle}$ of the clusters normalized by the dissipation scale of the carrier flow, as a function of $R_{\lambda}$. A typical cluster linear dimension ranging from $10 \eta$ to $20 \eta$ is found. When normalized by the dissipation scale of the flow, figure $5 \mathrm{c}$ shows that for $R_{\lambda} \leq 350$ the linear dimension of clusters exhibits a plateau around $11 \eta$. This is in quantitative agreement with the work by Aliseda and co-workers [1]. Working in passive grid turbulence in a wind tunnel with $R_{\lambda}=75$ and using a qualitative inspection they found that poly-dispersed water droplets form clusters with a typical area of $10 \eta$. For the largest Reynolds numbers (and Stokes numbers) investigated here, we observe an increase of the normalized clusters dimension (up to $20 \eta$ for the experiment at $R_{\lambda} \simeq 400$ ). The present data does not allow to discriminate wether this increase is a Reynolds number effect or a Stokes number effect. A possible explanation may be related to the fact that, in terms of Stokes number, the observed increase occurs above $S t \gtrsim 5$, hence above the optimal clustering Stokes number. The increase of the typical cluster size, may reveal that the decrease of clustering level occurs via an expansion (and hence a dilution) of the clusters. To better address this question, further measurements at even larger Reynolds numbers and with the possibility to adjust the droplets size (in order to vary $S t$ and $R_{\lambda}$ independently) will be carried in a new campaign of experiments.

Fractality of clusters. Finally we investigate the basic fractal properties of clustering by computing the joint histogram, for all clusters identified in a given experiment (here the experiment at $R_{\lambda} \simeq 300$, while the others experimental sets show similar tendencies), of the clusters perimeter $P_{C}$ and the root square of its area $\sqrt{A_{C}}$. This plot is shown in figure 9a. The figure shows many different tendencies 


\begin{tabular}{|c|c|c|}
\hline DNS resolution & $256^{3}$ & $512^{3}$ \\
\hline \hline$\nu$ & $7.6 \times 10^{-4}$ & $3 \times 10^{-4}$ \\
\hline$R_{\lambda}$ & 220 & 300 \\
\hline Pixel $(\mu \mathrm{m})$ & 110 & 80 \\
\hline $\mathrm{L}(\mathrm{cm})$ & 2.8 & 4.0 \\
\hline
\end{tabular}

Table 2: Parameters of simulations: numerical viscosity $\nu, R_{\lambda}$ (estimated using the same relations than the experimental value), grid point size in real world space and maximum lengthscale resolved in simulations.

but the fractional behavior of the exponent evidences the fractal nature of clusters with the presence of several different populations. Moreover, the almost continuum range for this exponent (ranging from $\sim 2$ to $\sim 3.5$ ) evidence again the extreme complexity of this structures.

Summary. We briefly summarize here the main experimental observations of clustering properties of inertial particles in turbulence, which will serve as elements of comparison with the numerical results presented in the next section :

- Small inertial particles tend to cluster, with Voronoï cells areas following a quasi-lognormal distribution.

- Clusters area PDFs exhibit a -2 power law for large areas and reveal a typical linear dimension of clusters of the order of $10 \eta$.

- Clusters exhibit a complex fractal structure.

\section{Numerical simulations.}

As mentioned in the introduction, our main goal here is to compare clustering properties of inertial particles in the experiment with that of specific topological points of a turbulent flow (in particular low acceleration and low vorticity points). The turbulent velocity field is obtained from Direct Numerical Simulations (DNS) of homogeneous isotropic turbulence. DNS has been performed using a pseudospectral code, with a resolution of $N=256^{3}$ and $512^{3}$. Further details of the code can be found in [11]. In order to warrant a proper resolution of all the turbulent scales it has been checked that $\kappa_{\eta} / \kappa_{\max } \sim 1$, where $\kappa_{\eta}=\eta^{-1}$ and $\kappa_{\max }$ is the maximum wave number resolved in the simulations. Table 3.1 shows the parameters of the simulations. It can be noted that $R_{\lambda}$ the for $256^{3}$ simulation is very close to that of the first experiment reported in table 1 while $R_{\lambda}$ for the $512^{3}$ simulation is very close to that of the third experiment in this same table. Comparison between numerics and experiments will therefore focus on this two specific experimental sets. To compare experimental and numerical scales, we choose here to match the dissipation scale of the DNS to that of this specific experiments. In this context the equivalent size in real world of a grid point and the maximum lengthscales resolved by the simulations can be deduced. In table 3.1 can be observed that the maximum lenghtscales resolved in simulations are in the order of a few centimeter and slightly smaller than the integral scale in experiments.

\subsection{Determination of "zero-acceleration" points.}

Let consider the Eulerian acceleration field of the flow at a given time (figure 6a). We define lowacceleration points as the set of points with an acceleration modulus below a certain threshold $a_{\text {thresh }}$. Strictly speaking this defines small patches of locally low-acceleration rather than actual zero-acceleration points. We point out though that results presented in this section have been shown to be robust to variations of the choice of the threshold used to define low-acceleration regions (sensitivity to the choice of threshold $a_{\text {thresh }}$ will be briefly discussed below) or if a Newton-Raphson method is used to actually find zeros of the acceleration field, as done in [7].

Then, considering that the thickness of the laser sheet in the experiment is of the order of $1 \mathrm{~mm}$, using the equivalent size of pixels in real world unit shown in table we consider stacks of slices of the numerical box to reproduce artificially a plane comparable to the experimental laser sheet (figure $6 \mathrm{~b}$ ). Zero acceleration points are then defined as the center of mass of the low acceleration patches on these 
(a)
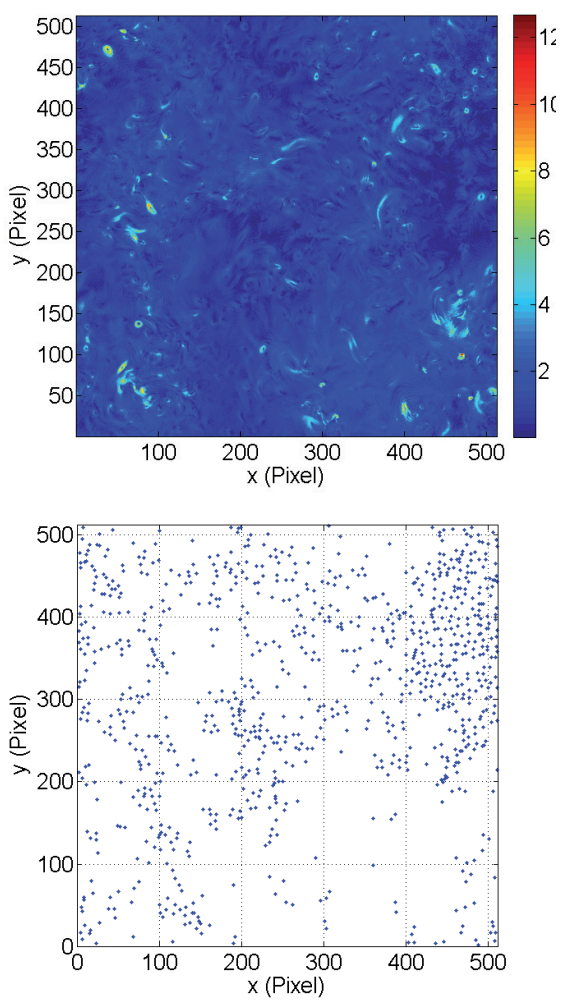

(c) (b)
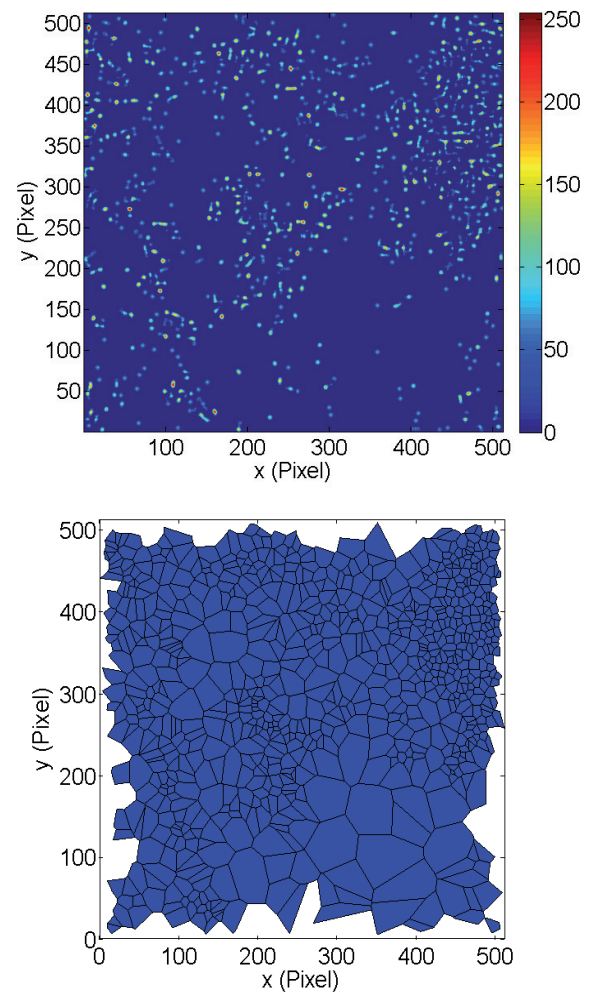

(c)

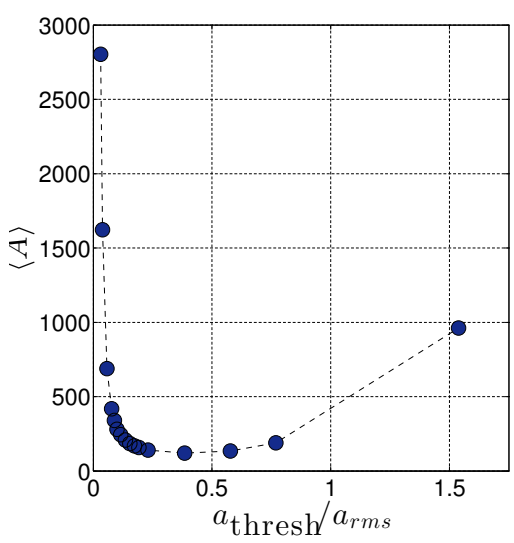

(e)

Figure 6: (a) Eulerian field of the modulus of the Lagrangian acceleration for a cross-section of $512 \times 512$ pixels in the DNS at $R_{\lambda} \simeq 300$. (b) Zero-acceleration patches (defined as regions with acceleration magnitude below a threshold $a_{\text {thresh }}=a_{r m s} / 10$ ) obtained after stacking 13 consecutive planes, corresponding to the thickness of the experimental laser sheet. (c) Zero-acceleration points obtained as the centers of mass of the patches in figure (b). (d) Voronoï tesselation of previous image. (e) Average Voronoï area as a function of the threshold $a_{\text {thresh }}$. The thresold has been taken at the very begining of the plateau. 


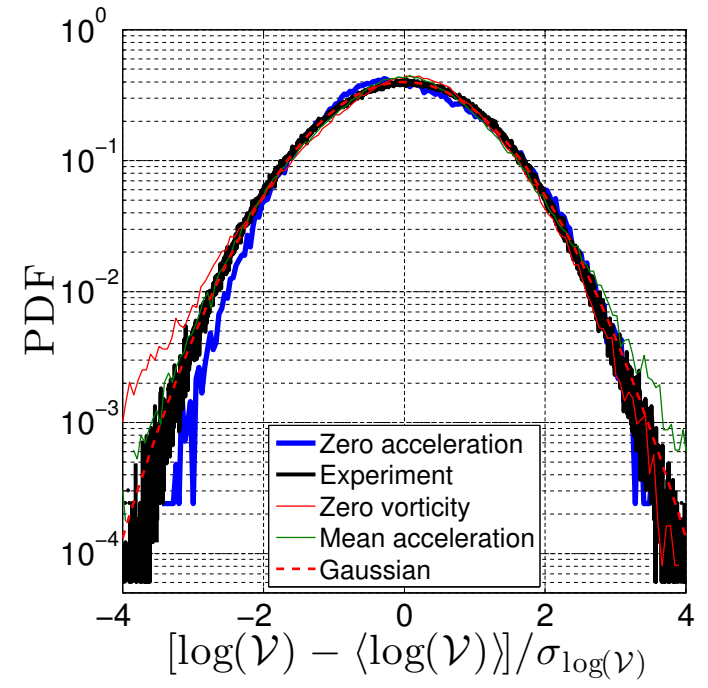

(a)

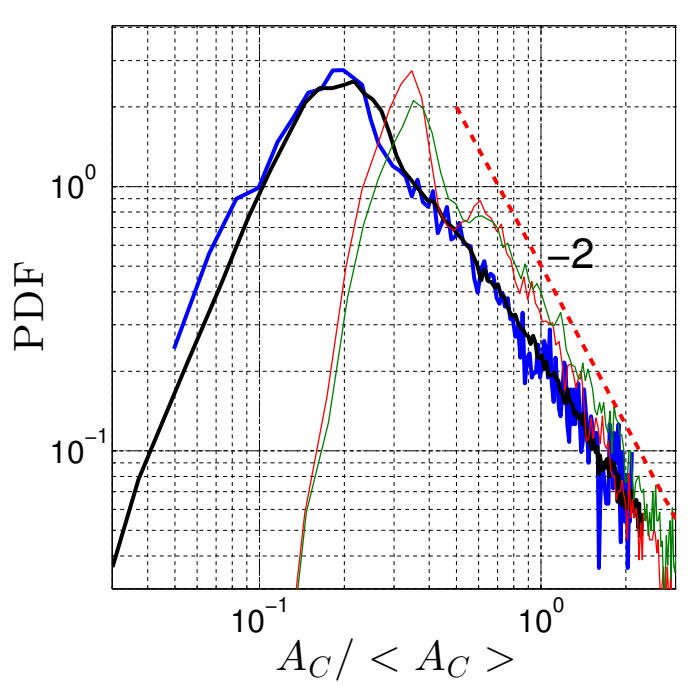

(b)

Figure 7: (a) Probability distribution function, centered and reduced, of $\log (\mathcal{V})$ obtained experimentally for $R_{\lambda}=220$ (black line) and numerically for zero-acceleration points (blue line), zero-vorticity points (red line) and mean-acceleration points (green line). The red dashed dashed line represents a Gaussian distribution. (b) Clusters area PDF obtained experimentally (black line) and numerically for zero-acceleration points (blue line), zero-vorticity points (red line) and mean-acceleration points (green line). The red dashed line emphasizes the asymptotic -2 power law.

reconstructed planes (figure 6c). Once these points are identified, the standard Voronoï analysis is performed (figure $6 \mathrm{~d}$ ).

The choice for the thresholding of acceleration modulus is done based on the average area $\langle A\rangle$ of the Voronoï cells of zero-acceleration points (which is the inverse of the global concentration of zeroacceleration points and hence directly reflects the number of zero-acceleration points detected). Figure $6 \mathrm{e}$ shows the dependency of $\langle A\rangle$ as a function of the threshold value $a_{\text {thresh }}$. It can be seen that when a strong criteria is employed $\left(a_{\text {thresh }}<<1\right)\langle A\rangle$ rapidly increases as very few zero-acceleration points are detected. the graph shows a clear plateau for thresholds in the range $0.1<a_{\text {thresh }} / a_{r m s}<0.8$. We have therefore used the minimum possible threshold within this plateau, hence taking $a_{\text {thresh }}$ equal to $10 \%$ of $a_{r m s}$.

\subsection{Voronoï analysis of zero-acceleration points.}

Log-normality. Figures $7 \mathrm{a}$ and $8 \mathrm{a}$ shows the $\mathrm{PDF}$ of $\log (\mathcal{V})$ (centered and reduced) for the zeroacceleration points in the DNS (blue line), for $R_{\lambda} \simeq 220$ and 300 respectively, compared to that of inertial particles in the experiment (black line). In both cases the agreement is very good. For $R_{\lambda} \simeq 300$ The similarity between both curves is remarkable. This observation indicates that zero-acceleration points do have a similar spatial distribution compared to inertial particles in the experiment. In particular, the quasi-lognormal shape of the $\mathrm{PDF}$ of $\log (\mathcal{V})$ (which was shown to be a robust feature of preferential concentration of particles $[12,15])$ is well reproduced by zero-acceleration points. Even the deviations to lognormality for the case at $R_{\lambda} \simeq 300$ are well captured.

Clusters geometry. To go further in the analysis, we investigate the geometry of clusters of zeroacceleration points and compare them to the experimental results for particles (already presented in the previous section). Figures $7 \mathrm{~b}$ and $8 \mathrm{~b}$ show the PDF of clusters area (normalized by the mean area) for the case of zero-acceleration points in DNS (blue line) and for that of inertial particles in the experiment (black line). Again the numerics reasonably reproduces the experimental results, specially for the lower $R_{\lambda}$. Although small clusters are slightly more represented for zero acceleration points than for the particles in the DNS at $N=512^{3}$, the algebraic tail with a -2 power law is robustly reproduced. The most probable normalized cluster area is very well captured by clusters of zero acceleration points for the 


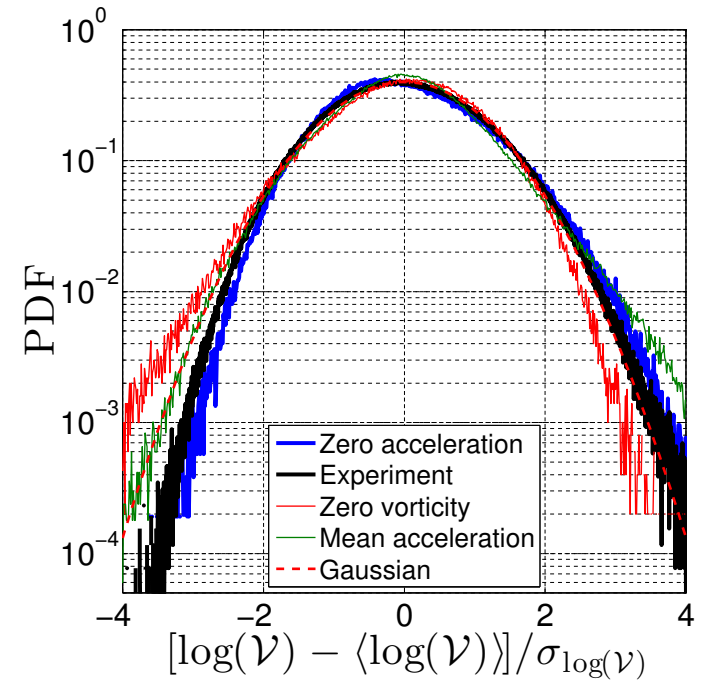

(a)

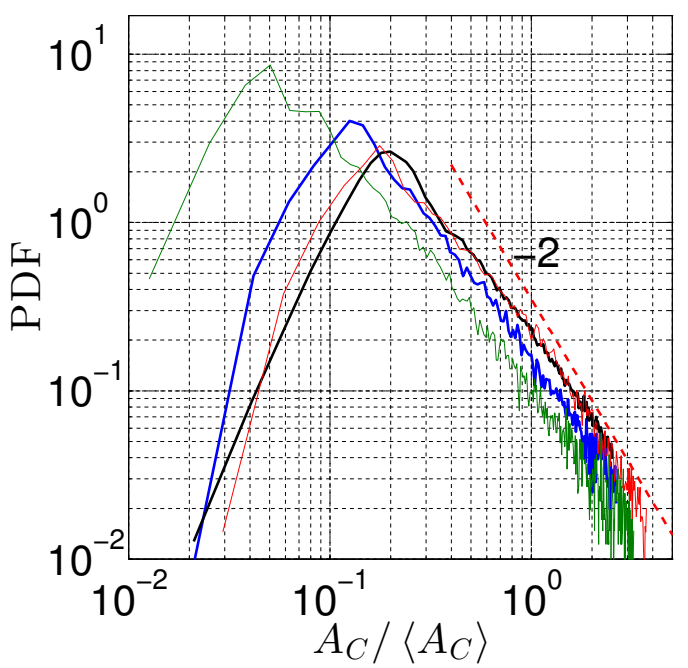

(b)

Figure 8: Same parameters shown in figure 7 for DNS at $R_{\lambda}=300$.

case at $R_{\lambda} \simeq 220$, and is slightly under-estimated in the simulation at $R_{\lambda} \simeq 300$. The typical linear size of zero-acceleration clusters is $\sqrt{\left\langle A_{C}\right\rangle}$ is of the order of $10 \eta$ in both cases (red circle in figure $5 \mathrm{c}$ ), hence very close to what has been found for actual particles in the experiment (figure 5c).

Clusters fractality. Finally, we show in figure $9 \mathrm{~b}$ the joint histograms of clusters of zero acceleration points and the square root of their area, to be compared to figure 9a, where the same quantities are plotted for the inertial particles in the experiment at $R_{\lambda} \simeq 300$. Both plots show similar tendencies, and evidence the fractal structure of both kind of clusters. The results for $R_{\lambda} \simeq 220$ exhibit the same tendencies.

\subsection{Voronoï analysis of other characteristic points of the turbulent field.}

In order to verify the actual relevance of zero-acceleration points to characterize clustering properties of inertial particles, other characteristic points of the flow have been investigated in the exact same manner, namely : the mean acceleration points (i.e. points where the acceleration is equal to the mean magnitude acceleration) and the zero-vorticity points. The first set of points is not related to any particular known clustering mechanisms, it is just a sort of placebo case which is not expected to have any particular relation with the experimental results for inertial particles. The second set of points on the contrary is related to the centrifugal mechanism, which predicts that heavy particles should explore preferentially low-vorticity regions as they are expelled outside the turbulent vortices. Interestingly, all these characteristic points are found to actually tend to clusterize, although clustering is significantly stronger for zero-acceleration points than for zero-vorticity or mean-acceleration points, as evidenced by the standard deviation $\sigma_{\mathcal{V}}$ of Voronoï cells, reported in Table 3. It can be seen in this table that for the simulation at $R_{\lambda} \simeq 220$ the clustering for zero-vorticity points is weak, as the standard deviation of Voronoï areas is 0.57 , hence only slightly higher than the value for a random Poisson process (for which we recall $\sigma_{\mathcal{V}} \simeq 0.53$ ). We also note that for both Reynolds numbers considered, the experimental value of $\sigma_{\mathcal{V}}$ is much closer to that of zero-acceleration points than to other points explored in the DNS.

Log-normality. The red and green lines in figures 7a and 8a show the centered and reduced PDFs of $\log (\mathcal{V})$ for zero-vorticity and mean-acceleration points respectively. For the $R_{\lambda} \simeq 220$ case, the PDF for zero-acceleration fits better the experimental PDF only marginally. However for $R_{\lambda} \simeq 300$ case, PFDs for zero-vorticity points and mean-acceleration points are in clear disagreement with that of particles in the experiment and zero-acceleration points are the only to conveniently reproduce the quasi log-normal distribution of Voronoï areas, as well as the deviations to log-normality. 


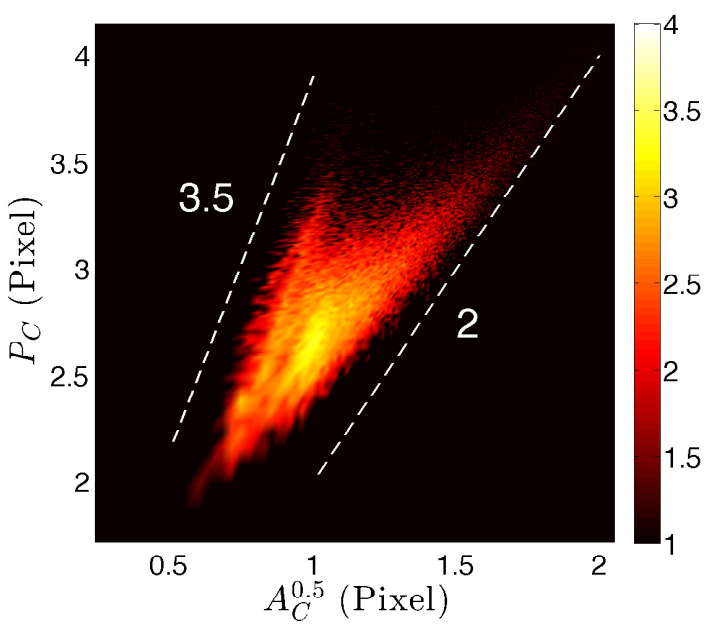

(a)

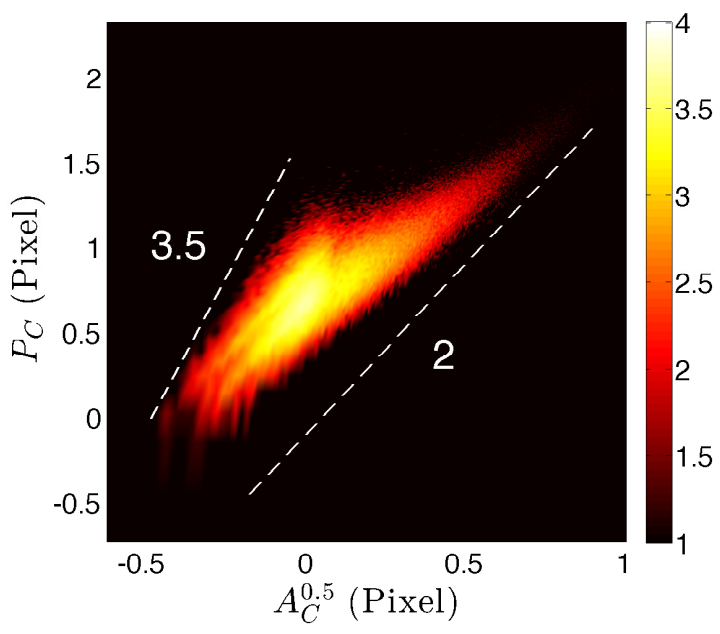

(b)

Figure 9: Joint histograms of clusters perimeter and the square root of their area obtained experimentally for particles (a) and numerically for zero-acceleration points (b). Both figures are in logarithmic scale.

\begin{tabular}{|c|c|c|c|c|}
\hline & Zero-acc. & Zero-vort. & Mean-acc. & Experiment \\
\hline \hline \multicolumn{5}{|c|}{$R_{\lambda} \simeq 220$} \\
\hline$\sigma_{\mathcal{V}}$ & 0.97 & 0.57 & 0.70 & 1.03 \\
\hline$\left\langle A_{C}\right\rangle$ & 8.5 & 4.5 & 4.5 & 10.5 \\
\hline \hline \multicolumn{5}{|c|}{$R_{\lambda} \simeq 300$} \\
\hline$\sigma_{\mathcal{V}}$ & 1.19 & 0.76 & 0.77 & 1.11 \\
\hline$\frac{\sqrt{\left\langle A_{C}\right\rangle}}{\eta}$ & 10.3 & 8.7 & 16.1 & 11.4 \\
\hline
\end{tabular}

Table 3: Standard deviation $\sigma_{\mathcal{V}}$ of Voronoï areas and typical linear cluster dimension (normalized by the dissipation scale $\eta$ ) for three different characteristic points of the velocity field in the DNS: zeroacceleration, mean-acceleration an zero-vorticity points. For comparison, the corresponding experimental values for particles in the wind-tunnel at each $R_{\lambda}$ are shown in the last column. 


\begin{tabular}{|c||c|c|c|c|}
\hline Points class & $\frac{\left|\sigma_{\mathcal{V}}^{\text {exp }}-\sigma_{\mathcal{V}}^{\text {num }}\right|}{\sigma_{\mathcal{V}}^{\text {exp }}}$ & Log-normality & $A_{c}$ PDF shape & $\frac{\sqrt{\left\langle A_{C}\right\rangle^{\text {exp }}}-\sqrt{\left\langle A_{C}\right\rangle^{\text {num }}}}{\sqrt{\left\langle A_{C}\right\rangle^{\text {exp }}}}$ \\
\hline \hline \multicolumn{5}{|c|}{$R_{\lambda} \simeq 220$} \\
\hline Zero-acc. & $6 \%$ & ++ & ++ & $19 \%$ \\
\hline Zero-vort. & $45 \%$ & + & - & $57 \%$ \\
\hline Mean-acc. & $32 \%$ & + & - & $57 \%$ \\
\hline \hline \multicolumn{5}{|c|}{$R_{\lambda} \simeq 300$} \\
\hline Zero-acc. & $7 \%$ & ++ & + & $10 \%$ \\
\hline Zero-vort. & $32 \%$ & - & ++ & $41 \%$ \\
\hline Mean-acc. & $31 \%$ & - & - & $24 \%$ \\
\hline
\end{tabular}

Table 4: Summary of the comparison between clustering properties of particles in experiments and the three different classes of special points investigated in the DNS. Two qualitative criteria are shown based on the Log-normality shape of the Voronoï areas PDFs (second column) and the ressemblance of clusters area PDF (third) column. "++", "+" and "-" indicate respectively "very good agreement", "reasonable agreement", "poor agreement". Two quantitative criteria are also given, based on the relative difference between experimental and numerical values for the variance of Voronoï areas $\sigma_{\mathcal{V}}$ (first columne) and the typical cluster size $\sqrt{\left\langle A_{C}\right\rangle}$ (last column). For each criteria, the best match between experiments and numerics has been highlighted in gray.

Clusters geometry. Finally, red and green lines in figures $7 \mathrm{~b}$ and $8 \mathrm{~b}$ show the PDF of clusters area for zero-vorticity and mean-acceleration points for the two Reynolds numbers considered. In all cases, mean-acceleration points disagree with experimental results, with a most probable normalized cluster area $\left(A_{C}^{\max } /\left\langle A_{C}\right\rangle\right)$ either larger or smaller than what is found for particles. The typical linear dimension of clusters of mean-acceleration points (upper triangles in figure 5c) does not match either that of particles in the experiment. Concerning zero-vorticity points figures $7 \mathrm{~b}$ and $8 \mathrm{~b}$ show that the PDF of clusters area also disagree with that of inertial particles in the experiment at $R_{\lambda} \simeq 220$, but reproduces surpringly well that of particles in the experiment at $R_{\lambda} \simeq 300$. However the normalized linear dimension of zero-vorticity clusters (down triangles in figure $5 \mathrm{c}$ ) systematically underestimates that of particles in the experiment.

\subsection{Summary}

Table 4 summarizes the main criteria previously discussed for the comparison of clustering properties between inertial particles in the experiment and the three different classes of special points of the carrier flow investigated from DNS data. We consider two qualitative criteria (based on the overall shape of the PDF of Voronoï cells areas and of the PDF of clusters area) and two quantitative criteria (based on the relative difference between experiments and numerics of the Voronoï areas variance $\sigma_{\mathcal{V}}$ and of the typical cluster linear dimension $\sqrt{\left\langle A_{C}\right\rangle}$.) Fractality is not shown in the table as all cases give similar fractal behaviors and therefore this does not seem to give a discriminatory criterion. The best match between experiments and numerics for each criterion has been highlighted in gray. It can be seen in Table 4 that, except for the shape of the PDF of clusters area in the simulation at $R_{\lambda} \simeq 300$ (for which zero-vorticity points give the best match), zero-acceleration points match best the experiment for all other cases. They also give a reasonable agreement for the shape of clusters PDF at $R_{\lambda} \simeq 300$. Put all together, these observations indicate that zero-acceleration points tend to organize in space in a very similar way as inertial particles do (in the range of Stokes and Reynolds numbers explored here).

\section{Discussion and conclusions}

We have presented in this work an experimental study of clustering of inertial particles in homogeneous isotropic turbulence at Reynolds numbers up to $R e_{\lambda} \sim 400$, and with particles Stokes numbers in the range [2-10]. A high level of clustering, analyzed by Voronoï tessellation analysis, has been observed. Statistics of Voronoï areas were found to follow a quasi-lognormal distribution, in agreement with previous studies at lower Reynolds numbers. We have identified clusters and analyzed some of their basic geometric properties. This study has revealed the existence of a characteristic size of clusters in the order of $10 \eta$, as well as a complex fractal structure of the clusters. Besides the simple characterization of preferential concentration of particles in turbulence, one goal of this work concerns the possibility to discriminate the relevance of several physical mechanisms possibly responsible for the clustering of particles, and 
more particularly the centrifugal effect (particles been expelled out of turbulent eddies) and the sweepstick mechanism (particles tending to stick to zero-acceleration points of the carrier turbulent field). These two mechanisms suggest that particles tend to preferentially sample specific points of the carrier flow, namely low vorticity points for the centrifugal effect and low acceleration points for the sweepstick mechanism. We have therefore investigated numerically the clustering properties of such points in isotropic homogeneous turbulence obtained from DNS at two Reynolds numbers $\left(R_{\lambda} \simeq 300\right.$ and $R_{\lambda} \simeq 220$ ). This study reveals that clustering of zero-acceleration points share many similarities with that of real particles in the experiments : (i) the quasi log-normality (including deviations to log-normality) of Voronoï areas, (ii) clusters area PDF and typical scale and (iii) fractality are very well captured by zero-acceleration points. It is also appealing that the value of $\sigma_{\mathcal{V}}$ (the standard deviation of Voronoï areas, which is an indicator of clustering level) for zero-acceleration points in DNS and particles in the experiment are very similar (as shown in table 3 ).

If we turn now the clustering properties of zero-vorticity points (relevant in a centrifugal scenario), our analysis reveals less clustering similarities compared to actual particles : (i) log-normality (and deviations from log-normality) are not well captured, (ii) clusters area PDF is well reproduced but clusters typical scale is underestimated (by $57 \%$ and $41 \%$ for the cases at $R_{\lambda} 220$ and $R_{\lambda} 300$ respectively, according to table 4), (iii) fractality is well reproduced. Besides, the clustering level of zero-vorticity points (given by $\sigma_{\mathcal{V}}$ ) is significantly less than that of the particles (45\% less in the simulation at $R_{\lambda} \simeq 220$ and $32 \%$ less in the simulation at $R_{\lambda} \simeq 300$, as shown in table 4 ).

As a test case, we have also considered the clustering properties of points of the carrier flow where acceleration amplitude is equal to the mean acceleration amplitude of the flow. These points, which are not expected to play any particular role does not reproduce satisfactorily any property of particles clustering (except the fractality, which seems to be present for all classes of clusters we have encountered).

Our conclusion is therefore that zero-acceleration points have clustering properties which are the closest to the particles experimentally investigated at the same $R_{\lambda}$. Although the observation that the spatial distribution of these two sets of points (zero-acceleration points and real particles) share similar statistical properties does not warrant that they actually coincide, it tends however to point toward a dominant role of stick-sweep effects in the clustering mechanism that play in the experiment. Future studies are planned in order to access simultaneous measurements in the experiment of both the particle concentration field and the velocity field of the carrier field in order to actually correlate both fields and corroborate the sweep-stick scenario. In these future studies, it will also be important to vary independently the Reynolds and Stokes numbers, in order to explore the influence of $S t$ at fixed $R_{\lambda}$ on clustering level and clustering mechanisms. As suggested numerically by Vassilicos and co-workers [3] using point particle models, centrifugal effect may be dominant for particles with $S t<1$ while sweepstick is expected to dominate for $S t>1$ (the case investigated in this work). Exploring such a transition between the two mechanisms may for instance help understanding the recent experimental results by Gibert et al. [6] who finds that the location of small and weakly inertial particles is correlated to low vorticity/strain dominated regions of the carrier field.

\section{Acknowledgements}

This work was supported by the French Agence Nationale pour la Recherche (project ANR-12-BS09011-03), the COST action on "Particles in turbulence" (project MP0806) and the French-Argentinian ECOS-Sud program (project A08U02).

\section{References}

[1] A Aliseda, A Cartellier, F Hainaux, and J C Lasheras. Effect of preferential concentration on the settling velocity of heavy particles in homogeneous isotropic turbulence. Journal of Fluid Mechanics, 468:77-105, 2002.

[2] Franz Aurenhammer. Voronoi diagrams - a survey of a fundamental geometric data structure. ACM Computing Surveys, 23:345-405, 1991.

[3] S W Coleman and J C Vassilicos. A unified sweep-stick mechanism to explain particle clustering in two- and three-dimensional homogeneous, isotropic turbulence. Physics of Fluids, 21(11):113301, November 2009. 
[4] Jr Fessler, Jd Kulick, and Jk Eaton. Preferential Concentration Of Heavy-Particles In A Turbulent Channel Flow. Physics of Fluids, 6(11):3742-3749, 1994.

[5] Renée Gatignol. The Faxen formulas for a rigid particle in an unsteady non-uniform Stoke flow. Journal de Mécanique Théorique et Appliquée, 2(2):143-160, 1983.

[6] Mathieu Gibert, Haitao Xu, and Eberhard Bodenschatz. Where do small, weakly inertial particles go in a turbulent flow? Journal of Fluid Mechanics, 698:160-167, March 2012.

[7] Susumu Goto and J C Vassilicos. Self-similar clustering of inertial particles and zero-acceleration points in fully developed two-dimensional turbulence. PHYSICS OF FLUIDS, 18(11), November 2006.

[8] Susumu Goto and J C Vassilicos. Sweep-Stick Mechanism of Heavy Particle Clustering in Fluid Turbulence. Physical Review Letters, 100(5):54503, 2008.

[9] Hideharu Makita. Realization of a large-scale turbulence field in a small wind tunnel. Fluid Dynamics Research, 8:53-64, 1991.

[10] Martin R Maxey and James J Riley. Equation of motion for a small rigid sphere in a nonuniform flow. Physics of Fluids, 26(4):883-889, 1983.

[11] Pablo D. Mininni, Duane Rosenberg, Raghu Reddy, and Annick Pouquet. A hybrid MPIOpenMP scheme for scalable parallel pseudospectral computations for fluid turbulence. Parallel Computing, $37(6): 316-326,2011$.

[12] R Monchaux, M Bourgoin, and A Cartellier. Preferential concentration of heavy particles: A Voronoi analysis. Physics of Fluids, 22(10):103304, 2010.

[13] Romain Monchaux, Mickael Bourgoin, and Alain Cartellier. Analyzing preferential concentration and clustering of inertial particles in turbulence. International Journal of Multiphase Flow, 40:1-18, 2012.

[14] Laurent Mydlarski and Zellman Warhaft. On the onset of high-Reynolds-number grid-generated wind tunnel turbulence. Journal of Fluid Mechanics, 320:331-368, 1996.

[15] Martin Obligado, Mahrane Missaoui, Romain Monchaux, Alain Cartellier, and Mickaël Bourgoin. Reynolds number influence on preferential concentration of heavy particles in turbulent flows. Journal of Physics: Conference Series, 318(5):052015, December 2011.

[16] C Poelma, J Westerweel, and G Ooms. Particle-fluid interactions in grid-generated turbulence. Journal of Fluid Mechanics, 589:315-351, 2007.

[17] R Poorte and A Biesheuvel. Experiments on the motion of gas bubbles in turbulence generated by an active grid. Journal of Fluid Mechanics, 461:127-154, 2002.

[18] Juan P L C Salazar, Jeremy de Jong, Lujie Cao, Scott H Woodward, Hui Meng, and Lance R Collins. Experimental and numerical investigation of inertial particle clustering in isotropic turbulence. Journal of Fluid Mechanics, 600:245-256, 2008.

[19] K D Squires and J K Eaton. Preferential concentration of particles by turbulence. Physics of Fluids A, 3(5):1169-1178, 1991 .

[20] Yoshiyuki Tagawa, Julián Martínez Mercado, Vivek N. Prakash, Enrico Calzavarini, Chao Sun, and Detlef Lohse. Three-dimensional Lagrangian Voronoï analysis for clustering of particles and bubbles in turbulence. Journal of Fluid Mechanics, 693:201-215, January 2012. 